\title{
Uncovering the Role of Key Active Site Side Chains in Catalysis: An Extended Brønsted Relationship for Substrate Deprotonation Catalysed by Wild-Type and Variants of Triosephosphate Isomerase
}

Yashraj S. Kulkarni ${ }^{1}$, Tina L. Amyes ${ }^{2}$, John Richard ${ }^{2}, *$ and S. C. L. Kamerlin ${ }^{1, *}$

1. Science for Life Laboratory, Department of Chemistry - BMC, Uppsala University, BMC Box 576, S-751 23 Uppsala, Sweden

2. Department of Chemistry, University at Buffalo, SUNY, Buffalo, New York 14260-3000, United States, Corresponding author email addresses: jrichard@buffalo.edu and lynn.kamerlin@kemi.uu.se 
Abstract: We report results of detailed empirical valence bond simulations that model the effect of several amino acid substitutions on the thermodynamic $\left(\Delta \mathrm{G}^{0}\right)$ and kinetic activation $\left(\Delta \mathrm{G}^{\ddagger}\right)$ barriers to deprotonation of dihydroxyacetone phosphate (DHAP) and D-glyceraldehyde 3phosphate (GAP) bound to wild-type triosephosphate isomerase (TIM), as well as to the K12G, E97A, K12G/E97A, I170A, I170A, I170A/L230A, and P166A variants of this enzyme. The EVB simulations model the observed effect of the P166A mutation on protein structure. The E97A, E97Q and E97D mutations of the conserved E97 side chain result in $\leq 1.0 \mathrm{kcal} \mathrm{mol}^{-1}$ decreases in the activation barrier for substrate deprotonation. The agreement between experimental and computed activation barriers is within $\pm 1 \mathrm{kcal} \mathrm{mol}^{-1}$, with a strong linear correlation between $\Delta \mathrm{G}^{\ddagger}$ and $\Delta \mathrm{G}^{0}$ for all 11 variants, with slopes $\beta=0.72\left(R^{2}=0.99\right)$ and $\beta=0.69\left(R^{2}=0.92\right)$ for the deprotonation of DHAP and GAP, respectively. These Brønsted-type correlations show that the amino acid side chains examined in this study function to reduce the thermodynamic driving force for deprotonation of the weakly $\alpha$-carbonyl carbon acid substrate to form the enediolate phosphate reaction intermediate. TIM utilizes the cationic side chain of K12 to provide direct electrostatic stabilization of the enolate oxyanion, and the nonpolar side chains of P166, I170A and L230A are utilized for the construction of an active site cavity that provides optimal stabilization of the enolate phosphate intermediate relative to the carbon acid substrate. 


\section{Introduction}

Triosephosphate isomerase (TIM) catalyzes the isomerization of $D$-glyceraldehyde 3phosphate to form dihydroxyacetone phosphate, by a proton transfer reaction mechanism through an enediolate reaction intermediate (Scheme 1). ${ }^{1-13}$ Proton transfer at carbon is ubiquitous in biology, and sets enzymes the difficult problem of reducing the large thermodynamic barriers to formation of carbanion intermediates of non-enzymatic reactions. ${ }^{14-16}$ This problem was solved by TIM approximately 3 billion years ago, ${ }^{17}$ through evolution of a catalyst that satisfies two criteria of perfection as outlined by Knowles in ref. ${ }^{18}$, and which uses the TIM-barrel protein fold present in $c a .10 \%$ of all enzymes. ${ }^{19}$ These observations have placed TIM at the forefront of enzymes targeted for mechanistic studies, because of the strong possibility that the results of studies on TIM will lead to conclusions that are broadly generalizable to other protein catalysts. ${ }^{20}$

Scheme 1. The mechanism of TIM-catalyzed isomerization of $D$-glyceraldehyde 3-phosphate (GAP) to form dihydroxyacetone phosphate (DHAP).

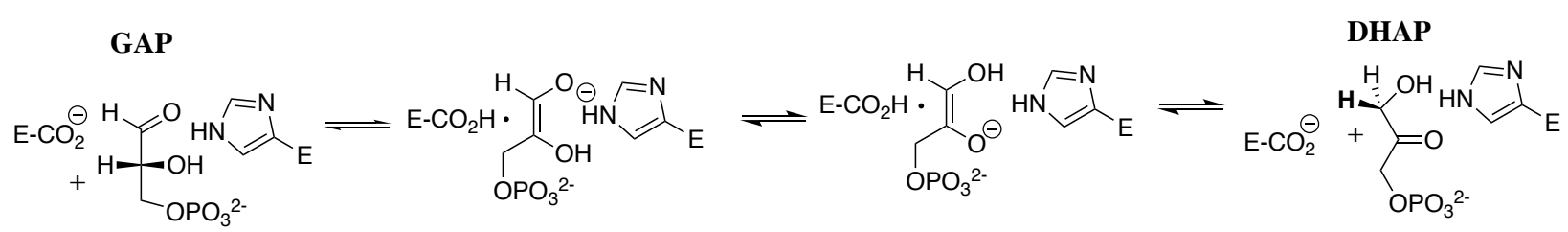

Computational studies on TIM have been pursued vigorously, ${ }^{3,4,7-11,13,21-37}$ because of their potential to provide a description of the events at the enzyme active site not accessible to examination by experimental studies. The many X-ray crystal structures for TIM from various organisms, and with a variety of ligands bound, ${ }^{38}$ provide valuable starting points for computational studies that model the experimental activation barrier for conversion of enzyme- 
bound substrate to product. It is important to test these models with larger bodies of experimental data in order to advance towards computational models that are able to fully reproduce the operation of TIM and other highly proficient enzymes. We have therefore challenged the empirical valence bond (EVB) $)^{39}$ approach to model the results of recent mutagenesis studies on $\mathrm{TIM},{ }^{14,40-44}$ with a focus on substitutions of the amino acid side chains shown in Figure 1 (using $y$ TIM numbering throughout this work).

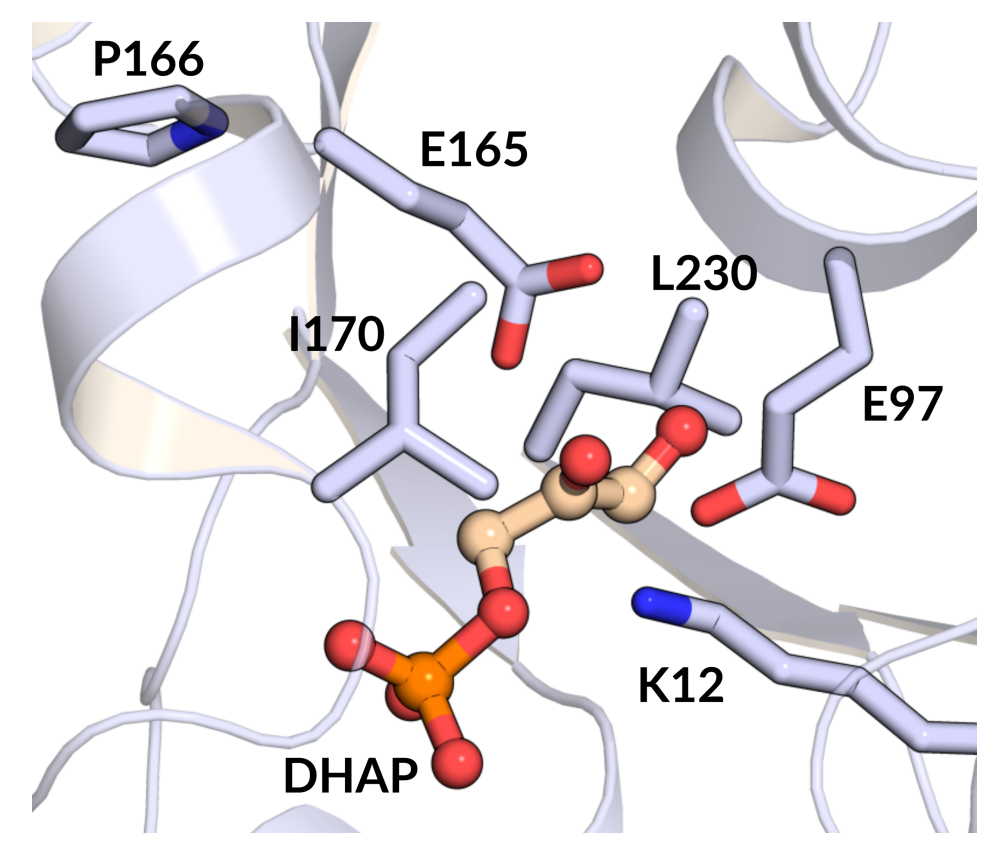

Figure 1. The X-ray crystal structure of the closed complex between yeast TIM ( $y$ TIM) and DHAP (PDB ID: $1 \mathrm{NEY}^{45}$ ), which shows the positions of the following active site side chains relative to the substrate DHAP: K12, E97, E165, P166, I170 and L230. In this study, we have examined single and/or double amino acid substitutions at each of these positions.

The residues of interest in this work are, specifically:

(1) The side chain cation of K12, which is positioned to interact with both the substrate phosphodianion and the partial negative charge that develops at the substrate carbonyl group at the transition state for substrate deprotonation (Scheme 1). ${ }^{41,46,47}$ The K12A substitution affects 
mainly the electrostatic stabilization of the anionic transition state,${ }^{47}$ and thus should be modelled with precision by EVB simulations.

(2) The highly conserved second-shell amino acid side chain anion of E97, ${ }^{48}$ which forms an ion pair to the cationic K12 side chain. ${ }^{44,46,47,49,50}$ The E97A substitution examines the modelling of replacement of a residue on the second-sphere of side chains at the enzyme active site. ${ }^{51}$

(3) The side chain of P166, which clashes with the carbonyl oxygen of G211 in loop 7 during the ligand driven movement of the loop from the unliganded open to the ligand-bound closed conformation of the loop. This steric clash results in movement of the E165 carboxylate from an initial "swung-out" position to a "swung-in" orientation that points into the TIM active site, where it is poised to deprotonate the enzyme-bound substrate. ${ }^{52-54}$ The P166A substitution eliminates this steric clash so that the E165 side chain remains in the swung-out position in the closed form of TIM. ${ }^{40}$ This amino acid substitution tests the precision of EVB simulations in modelling the effect of an amino acid perturbation that results in a defined change in protein structure.

(4) The side chains of I170 and L230, which fold over the catalytic carboxylate side chain of E165 at the closed form of TIM and displace to the bulk aqueous solvent four of six water molecules that lie within $6 \AA$ of this side chain. ${ }^{15,42,43,52,55}$ We previously reported the results of simulations to model the effect of I170A, L230A and I170A/L230A substitutions on the activation barrier for deprotonation of GAP and DHAP bound to yeast TIM ( $S c$ TIM), but report here slightly modified barriers obtained by the improved computational methods used in the present work. ${ }^{13}$ The I170A and L230A substitutions examine the precision of the EVB method in 
modelling the effect of a change in the polarity of the enzyme active site through replacement of hydrophobic side chains by solvent water. ${ }^{43}$

The EVB simulations do an excellent job of modeling the experimental effect of substitution of both the polar side chains of K12 and E97, and of the substitution of the nonpolar side chains of P166, I170 and L230, on the activation barriers $\left(\Delta \mathrm{G}^{\ddagger}\right)$ for deprotonation of enzyme-bound DHAP and GAP by the carboxylate side chain of E165. These simulations also provide values of $\Delta \mathrm{G}^{\circ}$ for proton transfer from the bound substrate to TIM to give the enediolate phosphate intermediate and the protonated carboxylic acid side chain, which cannot be determined by experiment. We report here a large extension of previously reported linear free energy relationships between the activation barriers $\Delta \mathrm{G}^{*}$ and Gibbs free energy change, $\Delta \mathrm{G}^{\mathrm{o}}$, for TIMcatalyzed deprotonation of enzyme-bound DHAP and GAP, to form the corresponding enediolate reaction intermediates, ${ }^{13}$ with Brønsted slopes of $\beta=0.72$ and 0.70 for the deprotonation of DHAP and GAP, respectively. This extended Brønsted relationship shows that the substituted polar and nonpolar side chains act cooperatively for the common purpose of minimizing the thermodynamic driving force for proton transfer from substrate to TIM, and that $70 \%$ of the change in the driving force for the reaction in water is expressed at the transition state for proton transfer. This change in reaction driving force is obtained through the direct stabilization of the enediolate phosphate by interaction with the cationic K12 side chain, and through the precise placement of the nonpolar P166, I170 and L230 side chains at a substrate cage, engineered by Nature with great precision in order to minimize the thermodynamic driving force for deprotonation of the enzyme bound substrate. 


\section{Methodology}

The empirical valence bond (EVB) approach ${ }^{39}$ was used in this work to model the TIMcatalyzed deprotonation of substrates DHAP and GAP, in wild-type and substituted TIM variants. We selected this approach based on our previous successes when using this model to capture the catalytic effect of wild-type TIM as well as of key active site substitutions on the rate-limiting deprotonation of these substrates. ${ }^{13}, 34,35,37$ All simulations in this study were performed using the OPLS-AA force field ${ }^{56}$ as implemented in the Q6 version of the Q simulation package, ${ }^{57,58}$ following the protocol outlined in detail in our previous studies. ${ }^{13,35}$ Our starting point for all EVB simulations was the crystal structure of wild-type yeast TIM ( $y$ TIM) in complex with DHAP (PDB ID: $1 \mathrm{NEY}^{45,59}$ ). In the case of the TIM variants studied in this work, all glycine and alanine substitutions were generated by simple truncation of the appropriate side chains, whereas in the case of the E97D and E97Q substitutions, the rotamers for the aspartate and glutamine side chains, respectively, were selected from the Dunbrack 2010 backboneindependent rotamer library, ${ }^{60}$ as implemented into Chimera. ${ }^{61}$ Simulations were performed on wild-type TIM, as well as on the K12G, E97A, K12G/E97A, E97D, E97Q, P166A, I170A, L230A and I170A/L230A variants.

All starting structures were then solvated in a $24 \AA$ spherical droplet of TIP3P water molecules, ${ }^{62}$ centered on the $\mathrm{C} 1$ atom of bound DHAP or GAP (see ref. ${ }^{13}$ for atom numbering), described using the Surface Constrained All Atom Solvent (SCAAS) approach. ${ }^{63}$ All residues within the inner $85 \%$ of this sphere were fully mobile, whereas those in the outer $15 \%$ were subjected to a $10 \mathrm{kcal} \mathrm{mol}^{-1} \AA^{-2}$ harmonic positional restraint to restrain them to their original crystallographic positions, and all residues outside the droplet were essentially fixed to their crystallographic positions using a $200 \mathrm{kcal} \mathrm{mol}^{-1} \AA^{-2}$ restraint, as described in previous work. ${ }^{13}$, 
34, 35, 37 All residues within the mobile region were kept in their expected ionization states at physiological $\mathrm{pH}$, assigned based on a combination of analysis using PROPKA $3.1^{64}$ and visual inspection, and residues outside the mobile region were kept in their neutral form, as is standard practice in such simulations in order to avoid system instabilities introduced due to the presence of charges outside the explicit simulation sphere. For a full list of ionized residues and histidine protonation states in our simulations, see Table $\mathbf{S 1 .}$

Each enzyme-substrate system was then subjected to six initial equilibration runs of $40 \mathrm{~ns}$ at $300 \mathrm{~K}$ with different initial velocities, heating from 0 to $300 \mathrm{~K}$ as described in refs. ${ }^{13,35}$ (Figures S1 and S2). This was then followed by five EVB simulations per equilibration run, performed using 51 mapping frames of $200 \mathrm{ps}$ in length each, resulting in a total of 30 individual EVB trajectories for each system, and 660 EVB trajectories over all systems. The starting points for the EVB simulations were generated by assigning new random seeds at the end point of the preceding equilibration run, and performing a further $110 \mathrm{ps}$ of equilibration to generate the new starting structures for the EVB simulations. All equilibration and EVB simulations were performed at the transition state for the proton transfer reaction, based on the valence bond states shown in Figure S3, as described in our previous work, ${ }^{13}$ in order to enable simultaneous propagation of EVB trajectories downhill towards the reactant and the product state. All simulations were performed using a $1 \mathrm{fs}$ timestep, to a total equilibration time of $240 \mathrm{~ns}$ per system $(5.28 \mu$ s over all systems $)$ and a total EVB simulation time of 306 ns per system (6.732 $\mu$ s over all systems). Note that we used the same EVB parameters $\left(H_{\mathrm{ij}}\right.$ and $\left.\alpha\right)$ as in our prior studies to describe the deprotonation of substrates DHAP and GAP. These parameters can be found in Table S2. For a description of these parameters, see ref. ${ }^{39,65}$, and for a description of how the EVB fitting was performed for these reactions see ref. ${ }^{13}$. All EVB parameters used in 
this work can be found in the Supporting Information of ref. ${ }^{13}$, and full protocols and simulation details can be found in our previous work on TIM. ${ }^{13,35}$

All energy analysis in this study was performed using the QCalc module of the Q simulation package. VMD version 1.9.1 ${ }^{66}$ was used to perform all structural analysis. Representative structures at various stationary points for the TIM-catalyzed deprotonation of DHAP and GAP in wild-type and P166A TIM were obtained by performing clustering analysis, using the method of Daura et al. ${ }^{67}$ as implemented in GROMACS version 4.6.5. ${ }^{68}$ A cutoff distance of $0.5 \AA$ was used for the analysis, which was based on structures sampled every 2 ps of the simulation time. All structural figures were generated using the PyMOL version 2.2.3 visualization package. ${ }^{69}$

\section{Results}

Figure 2 shows representative structures of the Michaelis complexes, transition states, and enediolate phosphate intermediates for the deprotonation of substrates DHAP and GAP by wildtype $y$ TIM during our EVB simulations. These simulations are performed starting with the highresolution structure of $y$ TIM (PDB ID: $1 \mathrm{NEY}^{45,59}$ ), and we therefore use the numbering for yTIM for all amino acid side chains. Table 1 summarizes the activation barriers and reaction free energies for the deprotonation of substrates DHAP and GAP to form enediolate phosphate intermediates (Scheme 1) catalyzed by the wild-type and substituted variants of TIM that were calculated in EVB simulations and comparisons between calculated activation barriers and activation barriers determined by experiment. The bar graphs from Figure 3 compare the calculated and experimental activation barriers. We note that, in several cases, calculated activation barriers are compared to experimental values obtained using enzyme kinetic parameters determined for amino acid substituted variants of TbbTIM. There is strong evidence 
that the active site catalytic side chains at TIMs from different organisms afford a similar stabilization of the isomerization transition state: (A) The active sites determined by X-ray crystallographic analysis of TIMs with as little as 50\% sequence homology are superimposable. ${ }^{70}$ (B) The similar kinetic parameters determined for wild-type TIMs from Trypanosomes, ${ }^{70}$ yeast ${ }^{41,71}$ rabbit muscle,${ }^{70}$ and chicken muscle, ${ }^{72}$ show that these enzymes provide a similar $( \pm 1$ $\mathrm{kcal} \mathrm{mol}^{-1}$ ) total stabilization of the rate-determining transition states for isomerization of GAP and DHAP. These results predict similar effects of side chain substitutions on the activation barrier.

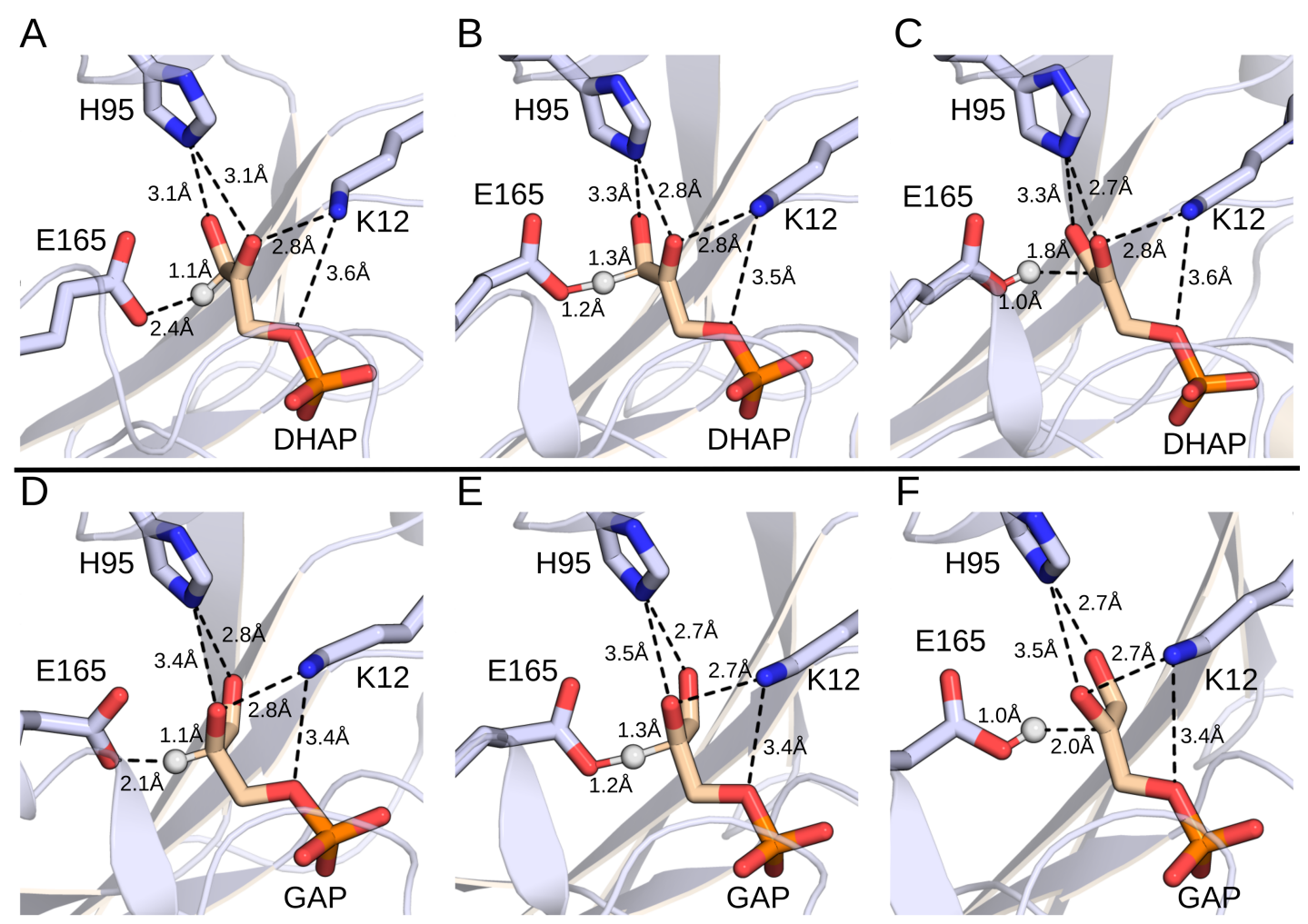

Figure 2. Representative structures of wild-type TIM at various stationary points. (A,D) Michaelis complexes, (B,E) transition states, and (C,F) intermediate states for the deprotonation of substrates (A-C) DHAP and (D-F) GAP. Shown here are the (donor-hydrogen and acceptor-hydrogen) distances involved in the proton transfer step, as well as distances between the side chains of the key active site residues H95 and K12 and the substrate. All distances annotated on this figure are averages over the entire simulation trajectory. The corresponding average distances for wild-type TIM and all the variants included in this study are presented in Tables S3 to S5. 
Table 1. Activation barriers and reaction free energies for the deprotonation of DHAP and GAP by different TIM variants, to form enediolate phosphate reaction intermediates. ${ }^{\mathrm{a}}$

\begin{tabular}{|c|c|c|c|c|c|c|c|c|}
\hline Substrate & Catalyst & $\Delta \mathrm{G}_{\exp }^{\ddagger}$ & $\Delta \mathrm{G}_{\text {calc }}^{\ddagger}$ & $\begin{array}{c}\Delta \mathrm{G}_{\mathrm{TIM}}^{\ddagger}- \\
\Delta \mathrm{G}_{\mathrm{non}}^{\ddagger}\end{array}$ & $\begin{array}{l}\Delta \mathrm{G}_{\mathrm{WT}}^{\ddagger}- \\
\Delta \mathrm{G}_{\mathrm{mut}}^{\ddagger}\end{array}$ & $\begin{array}{l}\mathrm{G}_{\text {calc }}^{\ddagger} \\
\Delta \mathrm{G}_{\text {exp }}^{\ddagger}\end{array}$ & $\Delta \mathrm{G}_{\text {calc }}^{\mathrm{o}}$ & $\begin{array}{c}\Delta \mathrm{G}_{\mathrm{TIM}^{-}}^{\mathrm{o}} \\
\Delta \mathrm{G}_{\text {non }}^{\mathrm{o}}\end{array}$ \\
\hline \multirow{11}{*}{ DHAP } & $\mathrm{CH}_{3} \mathrm{CH}_{2} \mathrm{CO}_{2}^{-}$ & & $25.4 \pm 0.03$ & & & & $19.0 \pm 0.03$ & \\
\hline & WT-TIM & $13.7^{\mathrm{b}, \mathrm{c}}$ & $15.0 \pm 0.4$ & -10.4 & & 1.3 & $5.2 \pm 0.4$ & -13.8 \\
\hline & I170A & $15.8^{\mathrm{b}}$ & $15.6 \pm 0.5$ & -9.8 & -0.6 & -0.2 & $5.8 \pm 0.7$ & -13.2 \\
\hline & L230A & $16.6^{\mathrm{b}}$ & $15.8 \pm 0.3$ & -9.6 & -0.8 & -0.8 & $7.0 \pm 0.4$ & -12.0 \\
\hline & $\mathrm{I} 170 \mathrm{~A} / \mathrm{L} 230 \mathrm{~A}$ & $17.4^{\mathrm{b}}$ & $16.8 \pm 0.2$ & -8.6 & -1.8 & -0.6 & $7.5 \pm 0.3$ & -11.5 \\
\hline & $\mathrm{K} 12 \mathrm{G}$ & & $18.7 \pm 0.3$ & -6.7 & -3.7 & & $10.5 \pm 0.5$ & -8.5 \\
\hline & E97A & $13.9^{\mathrm{b}}\left(14.1^{\mathrm{c}}\right)$ & $14.8 \pm 0.4$ & -10.6 & 0.2 & -0.9 & $4.8 \pm 0.5$ & -14.2 \\
\hline & K12G/E97A & & $18.9 \pm 0.3$ & -6.5 & -3.9 & & $10.6 \pm 0.4$ & -8.4 \\
\hline & E97D & $13.9^{\mathrm{b}, \mathrm{c}}$ & $14.9 \pm 0.3$ & -10.5 & 0.1 & 1.0 & $4.0 \pm 0.3$ & -15.0 \\
\hline & E97Q & $14.1^{\mathrm{b}}\left(14.5^{\mathrm{c}}\right)$ & $14.9 \pm 0.3$ & -10.5 & 0.1 & 0.8 & $5.2 \pm 0.6$ & -13.8 \\
\hline & P166A & $16.3^{\mathrm{b}}$ & $17.2 \pm 0.3$ & -8.2 & -2.2 & 0.1 & $8.5 \pm 0.3$ & -10.5 \\
\hline \multirow{11}{*}{ GAP } & $\mathrm{CH}_{3} \mathrm{CH}_{2} \mathrm{CO}_{2}^{-}$ & & $24.0 \pm 0.03$ & & & & $16.0 \pm 0.04$ & \\
\hline & WT-TIM & $12.9^{\mathrm{b}}\left(12.5^{\mathrm{d}}\right)$ & $13.7 \pm 0.5$ & -10.3 & & 0.8 & $2.6 \pm 0.6$ & -13.4 \\
\hline & I170A & $16.0^{\mathrm{b}}$ & $15.5 \pm 0.7$ & -8.5 & -1.8 & -0.5 & $4.1 \pm 0.7$ & -11.9 \\
\hline & L230A & $14.2^{\mathrm{b}}$ & $14.4 \pm 0.3$ & -9.6 & -0.7 & 0.2 & $3.3 \pm 0.4$ & -12.7 \\
\hline & I170A/L230A & $16.3^{\mathrm{b}}$ & $15.2 \pm 0.3$ & -8.8 & -1.5 & -1.1 & $4.3 \pm 0.5$ & -11.6 \\
\hline & $\mathrm{K} 12 \mathrm{G}$ & $17.7^{\mathrm{c}}$ & $16.2 \pm 0.4$ & -7.8 & -2.5 & -1.5 & $6.0 \pm 0.4$ & -10.0 \\
\hline & E97A & & $13.7 \pm 0.7$ & -10.3 & 0.0 & & $1.7 \pm 0.7$ & -14.3 \\
\hline & K12G/E97A & & $16.5 \pm 0.4$ & -7.5 & -2.8 & & $6.5 \pm 0.4$ & -9.5 \\
\hline & E97D & $15.2^{\mathrm{d}}$ & $13.0 \pm 0.4$ & -11.0 & 0.7 & -2.2 & $1.5 \pm 0.6$ & -14.5 \\
\hline & E97Q & $17.4^{\mathrm{d}}$ & $14.4 \pm 0.4$ & -9.6 & -0.7 & -3.0 & $3.4 \pm 0.4$ & -12.6 \\
\hline & P166A & $15.6^{\mathrm{b}}$ & $16.4 \pm 0.4$ & -7.6 & -2.7 & 0.1 & $5.4 \pm 0.4$ & -10.6 \\
\hline
\end{tabular}

${ }^{\text {a }}$ All energies are shown in $\mathrm{kcal} \mathrm{mol}^{-1}$. Calculated energies are averages and standard error of the mean over 30 individual EVB trajectories, as described in the Methodology section. Shown here are both the values calculated in aqueous solution $\left(\mathrm{CH}_{3} \mathrm{CH}_{2} \mathrm{CO}_{2}{ }^{-}\right.$as the general base) and in different TIM variants. The corresponding experimental 
values are based on kinetic data for different TIM variants $\left({ }^{\mathrm{b}}\right.$ denotes data from TbbTIM, ${ }^{\mathrm{c}}$ denotes data from $y$ TIM and $^{\mathrm{d}}$ denotes data from PfTIM), where available, as presented in refs. ${ }^{14,40-44,49,70} . \Delta \mathrm{G}^{\ddagger}$ calc and $\Delta \mathrm{G}_{\text {exp }}^{\ddagger}$ denote the calculated and experimental activation free energies, respectively, $\Delta \mathrm{G}^{0}$ calc denotes the calculated reaction free energy, $\Delta \mathrm{G}^{\ddagger}$ TIM $-\Delta \mathrm{G}^{\ddagger}$ non denotes the difference between the activation free energy calculated for the TIM-catalyzed reaction and for the background reaction in aqueous solution, $\Delta \mathrm{G}^{*} \mathrm{wT}-\Delta \mathrm{G}^{\dagger}$ mut denotes the difference between the activation free energy calculated for wild-type TIM and for the different TIM variants considered in this work, $\Delta \mathrm{G}^{\ddagger}$ calc- $\Delta \mathrm{G}_{\text {exp }}^{\ddagger}$ denotes the difference between the calculated and experimental activation free energies for wild-type TIM and the different TIM variants considered in this work, and $\Delta \mathrm{G}^{0}{ }_{\text {TIM }}-\Delta \mathrm{G}^{0}{ }_{\text {non }}$ denotes the difference between the reaction free energy calculated for different TIM variants and for the background reaction in aqueous solution.
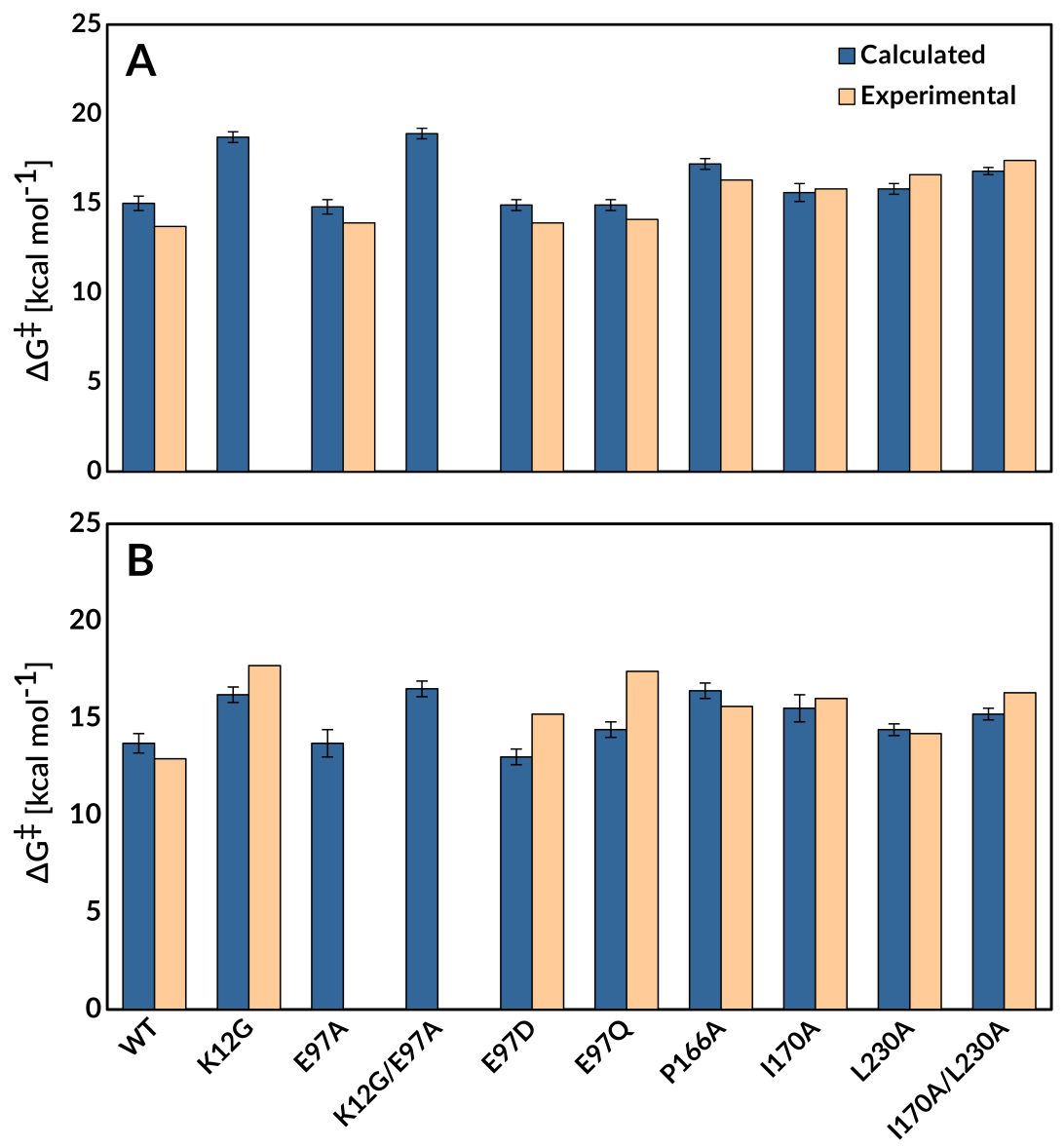

Figure 3. A comparison of the activation free energies determined by EVB simulations (blue), and from experimental kinetic parameters (based on experimental kinetic measurements performed on TbbTIM, $y$ TIM and PfTIM ${ }^{14,40-44,70}$, tan), for TIM-catalyzed deprotonation of substrates (A) DHAP and (B) GAP to form enediolate reaction intermediates. The raw data for this figure is presented in Table $\mathbf{1}$. 


\section{Discussion}

The activation barriers reported in Table $\mathbf{1}$ and Figure $\mathbf{3}$ were obtained from EVB simulations performed starting with the high-resolution structure of $y$ TIM (PDB ID: 1NEY ${ }^{45,59}$ ). The overall agreement between the experimental and computed activation barriers is generally within $\pm 1 \mathrm{kcal} \mathrm{mol}^{-1}$, and is similar to the previously published agreement between experimentally and computationally determined effects, respectively, of I170A, L230A, and I170A/L230A substitutions on the activation barriers for TbbTIM- and $y$ TIM-catalyzed deprotonation of GAP and DHAP. ${ }^{13}$ We have increased the radius of the water droplet/mobile region in our EVB simulations from $20 \AA$ in our previous computational studies of TIM and its variants $^{13,35}$ to $24 \AA$ in the present work. ${ }^{37}$ This improves the description of TIM flexibility through the use of a larger mobile region, but comes with a greater computational cost associated with the increased size of the water droplet. The $4 \AA$ increase in the radius of the water droplet (and thus also the size of the mobile region in the EVB simulations) in the present study results in up to a $0.8 \mathrm{kcal} \mathrm{mol}^{-1}$ higher calculated activation barriers for the deprotonation of both DHAP and GAP. However, there is no discernible improvement in the agreement between the experimental and calculated activation barriers for wild-type TIM and the I170A, L230A I170A/L230A variants that were previously modeled using a $20 \AA$ radius for the water droplet/mobile region. ${ }^{13}$ This is consistent with a diminishing return in computational precision for these EVB simulations, from the increasing computational cost associated with increases in the radius of the water droplet/mobile region to $>20 \AA$.

Protein Variants. K12 and E97 Substitutions. The K12M/G15A variant of TIM showed no detectable activity for catalysis of isomerization, ${ }^{47}$ and the X-ray crystal structure for this variant revealed that the interaction between the K12 side chain and the substrate phosphodianion is 
required to observe substrate bound to the crystalline protein. ${ }^{73}$ The $\mathrm{K} 12 \mathrm{G}$ variant of $y \mathrm{TIM}$ is active, but the amino acid substitution results in a $7.8 \mathrm{kcal} \mathrm{mol}^{-1}$ increase in the activation barrier to $k_{\text {cat }} / K_{\mathrm{m}}$ for TIM-catalyzed isomerization, ${ }^{41}$ and much of the lost catalytic activity may be rescued by alkyl ammonium cations. ${ }^{46}$ The interaction between GAP and K12G $y$ TIM is too weak to provide clear evidence for saturation. ${ }^{41}$ However, the K12G substitution results in a 2.4 kcal mol-1 destabilization of the complex to the intermediate analog phosphoglycolate (PGA) that is consistent with a $2.4 \mathrm{kcal} \mathrm{mol}^{-1}$ destabilization of the Michaelis complex to GAP and a (7.8 2.4) $=5.4 \mathrm{kcal} \mathrm{mol}^{-1}$ increase in the barrier for conversion of this complex to the isomerization transition state. ${ }^{41}$

Our EVB simulations predict increases in the activation barrier to substrate deprotonation $\left(\Delta \Delta \mathrm{G}^{\ddagger} \mathrm{wt} \rightarrow\right.$ mut $)$ of $3.7 \mathrm{kcal} \mathrm{mol}^{-1}$ for substrate DHAP and $2.5 \mathrm{kcal} \mathrm{mol}^{-1}$ for substrate GAP, compared to wild-type TIM, upon truncation of the K12 side chain to a glycine. By comparison, we obtain similar -3.9 and $-2.9 \mathrm{kcal} \mathrm{mol}^{-1}$ electrostatic contributions to the calculated activation free energies for the deprotonation of substrates DHAP and GAP, respectively, by wild-type TIM (Figure 4), evaluated by applying the linear response approximation (LRA) ${ }^{74,} 75$ to the corresponding EVB trajectories. ${ }^{13,} 35$ The calculated 3 to $4 \mathrm{kcal} \mathrm{mol}^{-1}$ effect of the K12G substitution on the activation barrier to substrate deprotonation is smaller than the estimated 5.4 kcal mol-1 effect of this substitution on the activation barrier to isomerization of enzyme-bound GAP. However, there is a large uncertainty in the partitioning of the overall $7.8 \mathrm{kcal} \mathrm{mol}^{-1}$ increase in the experimental activation barrier into effects on $k_{\text {cat }}$ and $K_{\mathrm{m}}$.

The K12 side chain runs across the surface of TIM, and the side chain cation interacts with substrate bound beneath the protein surface. ${ }^{41,46,47,73}$ The E97 side chain lies in the second shell of active site side chains and forms an anchoring ion pair to the first shell $\mathrm{K} 12$ side chain cation 
(Figure 1). This side chain is conserved at the active sites of all TIMs. ${ }^{48}$ We have carried out EVB simulations to model the effects of amino acid substitutions of E97, in order to examine the functional role that mandates conservation of anchoring the K12•E97 ion pair at TIM.

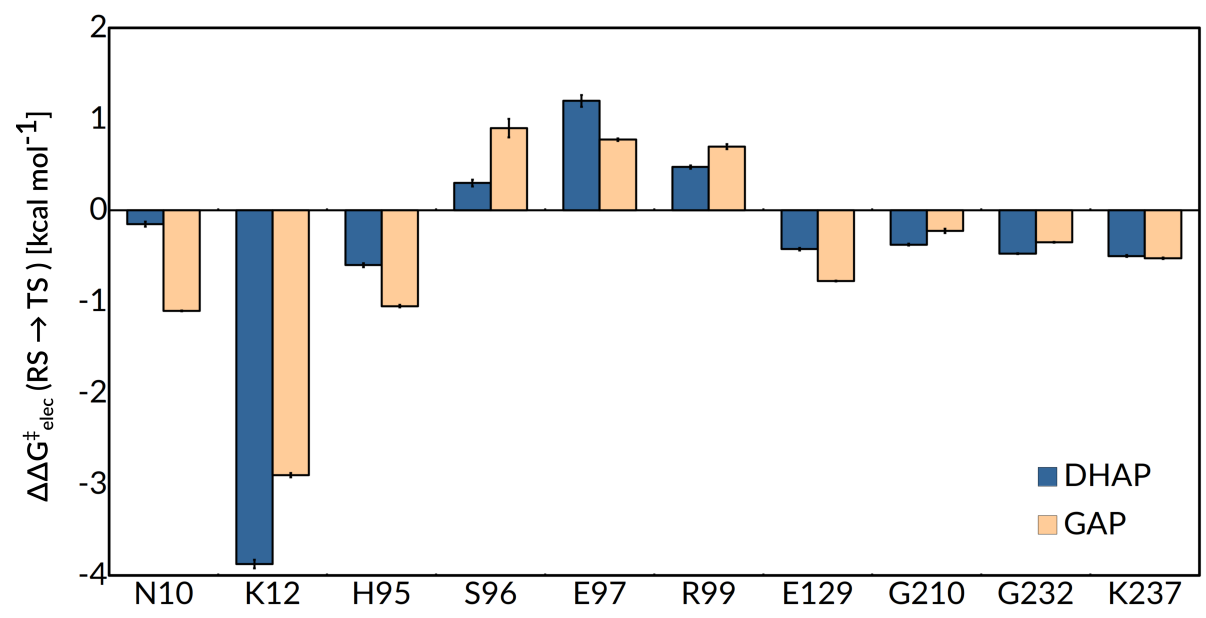

Figure 4. Electrostatic contributions of individual residues to the calculated activation free energies $\left(\Delta \mathrm{G}^{\star}\right.$ elec $)$ for the deprotonation of DHAP and GAP by wild-type TIM. As in our previous work, ${ }^{13,} 35$ all values were calculated by applying the linear response approximation ${ }^{74,75}$ to the calculated EVB trajectories, and scaled assuming a dielectric constant of 4 for the active site. All values shown are averages over 30 independent trajectories and the error bars represent the standard error of the mean. The corresponding raw data for this figure is shown in Table S6.

The E97A, E97D and E97Q substitutions of $y$ TIM each result in $\leq 0.7 \mathrm{kcal} \mathrm{mol}^{-1}$ changes in the calculated activation barriers for wild-type TIM-catalyzed deprotonation of DHAP and GAP (Table 1). These changes are within the uncertainty of these simulations and represent increases in the activation barrier, except for the $0.7 \mathrm{kcal} \mathrm{mol}^{-1}$ transition state stabilization calculated for the E97D variant of $y$ TIM. There is good agreement between the calculated effect of these three substitutions on the activation barrier for catalysis by wild-type $y$ TIM and the experimental activation barrier (where available) for catalysis by TbbTIM (Figure 3). ${ }^{44}$ The LRA simulations 
(Figure 4) are consistent with a $\sim 1 \mathrm{kcal} \mathrm{mol}^{-1}$ destabilizing contribution to the activation barrier for TIM-catalyzed deprotonation of DHAP and GAP from interactions between the side chain anion of E97 and the distant anionic transition state.

The experimental and computational results show that the contribution of the anchoring $\mathrm{K} 12 \cdot \mathrm{E} 97$ ion pair to the enzymatic rate acceleration is $<1 \mathrm{kcal} \mathrm{mol}^{-1}$. EVB simulations likewise show that the E97A substitution of K12G TIM to give the double K12G/E97A substitutions results in $\mathrm{a} \leq 0.3 \mathrm{kcal} \mathrm{mol}^{-1}$ increase in the calculated activation barriers for the K12G $y \mathrm{TIM}$ catalyzed deprotonation of DHAP and GAP, and that the effect of the K12G substitution on activation barrier for catalysis by the E97A variant lies within $0.3 \mathrm{kcal} \mathrm{mol}^{-1}$ of the effect of the K12G substitution on the activation barrier for catalysis by wild-type $y$ TIM. We conclude that the role of the E97 side chain in preorganization of the K12 side chain at a K12•E97 salt bridge $^{76-78}$ does not provide for strong stabilization of the isomerization transition state. This is consistent with the conclusion that the evolutionary pressure to optimize the activity of TIM, which plays a critical metabolic role in cellular energy production by glycolysis,${ }^{79}$ is so strong that the E97 side chain is strictly conserved in order to obtain a ca. $1 \mathrm{kcal} \mathrm{mol}^{-1}$ stabilization of the rate determining transition state. The E97 carboxylate anion is hydrogen bonded to the T75 side chain at the second subunit of TIM. ${ }^{80}$ The placement of this side chain in the middle of a network of hydrogen bonds that spans the K12 and T75 side chains at neighboring protein subunits implies an additional role for E97 in maintaining the dimeric structure for TIM, which merits investigation.

Finally, we note that the curious result that E97D and E97Q substitutions at TIM from Plasmodium falciparum (PfTIM) result in 4,000- and 100-fold decreases in $k_{\text {cat }}$ for PfTIMcatalyzed isomerization of GAP that are not modeled by our simulations of $y$ TIM. ${ }^{49}$ Schwans and 
coworkers have proposed an explanation for this organismal dependence in the measured kinetics, based on small differences in the structures of TbbTIM and PfTIM, for the larger effect of E97D and E97Q substitutions on $k_{\text {cat }}$ for catalysis by PfTIM compared with TbbTIM. ${ }^{44}$

Substitution of P166. The triad of nonpolar side chains of P166, I170 and L230 line the active-site cavity of TIM. The observation of falloffs in catalytic activity for P166A, ${ }^{40,81}$ I170A, ${ }^{15,}{ }^{42} \mathrm{~L}_{230 \mathrm{~A}},{ }^{55}$ and $\mathrm{I} 170 \mathrm{~A} / \mathrm{L} 230 \mathrm{~A}^{43}$ variants of TIM show that these side chains play a structural role in the enhancement of the reactivity of the carboxylate side chain of E165 for deprotonation of enzyme-bound substrate. The effects of I70A, L230A and I170A/L230A substitutions on the reaction activation barrier reflect global changes in the interactions between polar active-site groups and the enediolate-like transition state observed upon substitution of the hydrophobic side chains of I70A and L230A by water at the variant enzymes. ${ }^{43}$ We have previously used the EVB-method successfully to model the effects of I70A, L230A and I170A/L230A substitutions. ${ }^{13}$

Wierenga and coworkers have provided a detailed description of the role of the P166 side chain in driving the carboxylate side chain of E165 into a hydrophobic cage, where it is flanked by the hydrophobic side chains of I170 and L230 and lies proximal to the carbon acid substrate. ${ }^{82}$ They showed that the X-ray crystal structure of the P166A variant complexed to phosphoglycolate (PDB ID: $2 \mathrm{~J} 24^{40,59}$ ) is essentially superimposable on the wild-type complex to DHAP (PDB ID: $1 \mathrm{NEY}^{5,59}$ ), except that relief of the steric clash between the carbonyl oxygen of G209 and the pyrolidine side chain of P166 at the P166A variant allows the carboxylate side chain of E165 to relax to the "swung-out" position observed for unliganded TIM (Figure 5). ${ }^{40}$ This small structural change observed in the P166A variant is associated with ca. 50-fold decreases in $k_{\text {cat }}$ and 2-fold decreases in $K_{\mathrm{m}}$ for the turnover of GAP and DHAP. ${ }^{81}$ 

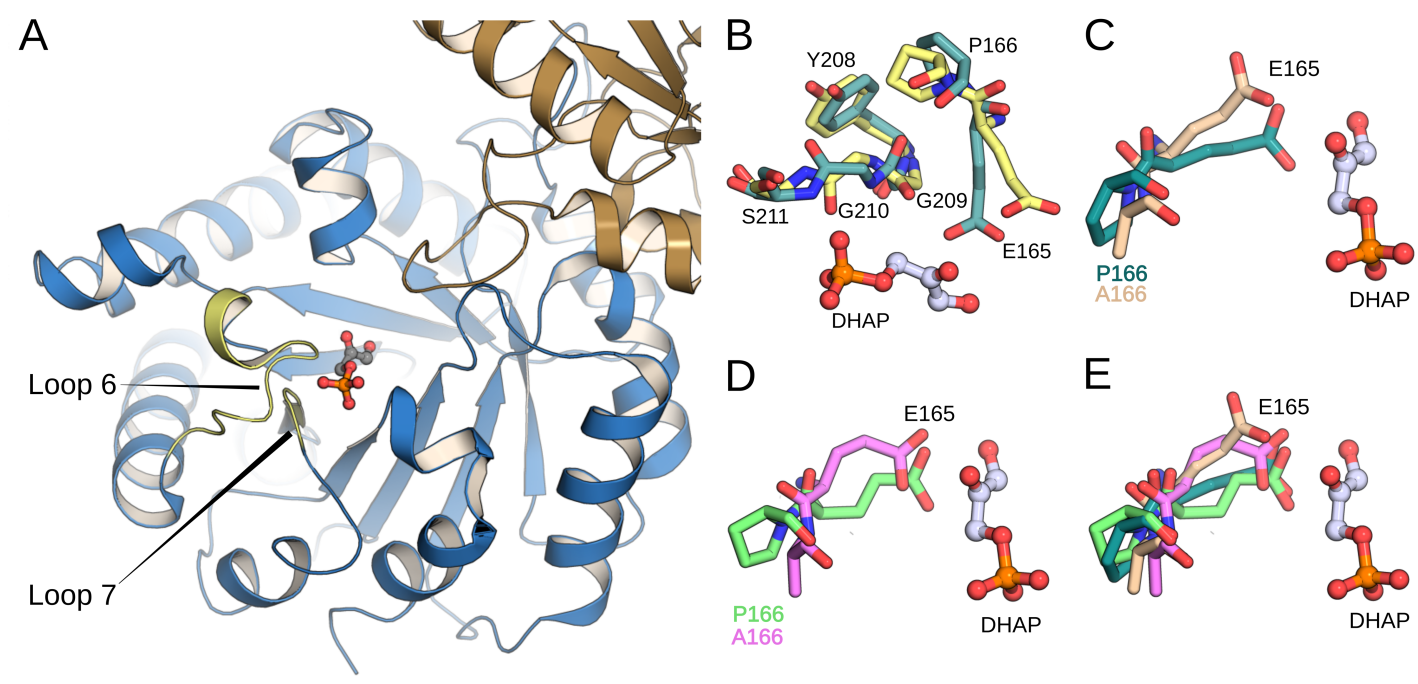

Figure 5. (A) Close-up view of the tertiary structure of yeast TIM ( $y$ TIM) complexed to DHAP (PDB ID: 1NEY5, ${ }^{59}$ ). (B) An overlay of X-ray structures of loop 7 in TIM structures with closed (PDB ID: $1 \mathrm{NEY}^{5,59}$ dark green) and open (PDB ID: 1YPI ${ }^{59,83}$, yellow) conformations of loop 6. (C) An overlay of X-ray structures showing the "swung in" and "swung out" conformations of E165 in wild-type $y$ TIM (PDB ID: 1NEY 5 , , dark green) and the complex of P166A TbbTIM (PDB ID: 2J2740, 59, tan). (D) An overlay of E165 in representative structures from our EVB simulations (obtained from clustering analysis as described in the Methodology section) at the Michaelis complexes wild-type (light green) and P166A TIM (pink). (E) Overlay of all structures shown in panels (C) and (D).

EVB simulations of the P166A variant were carried out, starting with the crystal structure of the $y \mathrm{TIM} \cdot \mathrm{DHAP}$ complex (PDB ID: $1 \mathrm{NEY}^{45,59}$ ), and substituting a $-\mathrm{CH}_{3}$ group for the P166 side chain. The carboxylate side chain of E165 lies initially in the "swung-in" position for wild-type TIM, but, despite starting with the wild-type crystal structure, these simulations remarkably show a similar relaxation in the position of this side chain to the "swung-out" position observed for the crystal structure of the P166A variant complexed to PGA (Figure 5).$^{40}$ These changes in structure result in small increases in the enzyme-substrate donor-acceptor distance from $3.0 \AA$ for the complexes between wild-type TIM and DHAP or GAP, to $3.3 \AA$ and $3.1 \AA$ for complexes between the P166A variant of TIM and DHAP (Table S3) or GAP (Table S4), respectively. 
The EVB simulations of the P166A variant of $y$ TIM give 2.7 and $2.2 \mathrm{kcal} \mathrm{mol}^{-1}$ increases in the activation barrier for deprotonation of GAP and DHAP, respectively, that are in good agreement with the 2.6 and $2.3 \mathrm{kcal} \mathrm{mol}^{-1}$ increases in activation barriers determined from the effect of the substitution on $k_{\text {cat }}$ for isomerization reactions catalyzed by TbbTIM. ${ }^{81}$ We conclude that these simulations model both the effect of the P166A substitution on the structure of the enzyme-ligand complex, and on the activation barrier for TIM-catalyzed deprotonation of enzyme-bound substrate.

\section{Linear Free Energy Relationships}

We have reported good linear correlation between the kinetic (activation free energy, $\Delta \mathrm{G}^{\ddagger}$ ) and thermodynamic (reaction free energy, $\Delta \mathrm{G}^{0}$ ) barriers for the deprotonation of substrates DHAP and GAP by propionate ion from solvent, wild-type TIM, and the I170A, L230A and I170A/L230A variants. ${ }^{13}$ Figure 6 shows large extensions of these linear free energy relationships (LFER) to include all 11 enzyme variants studied in this work. These LFER define strong linear correlations between the kinetic and thermodynamic reaction barriers, with slopes of $\beta=0.72\left(R^{2}=0.99\right)$ and $\beta=0.70\left(R^{2}=0.92\right)$ for the deprotonation of DHAP and GAP, respectively. The better $R^{2}$ value observed for deprotonation of DHAP reflects the use of the same ground-state protein structure for the TIM-DHAP complex in calculation of both sets of reaction barriers. ${ }^{45}$ There are no crystal structures for the TIM-GAP complex, which presumably undergoes isomerization to the thermodynamically favored TIM-DHAP complex. ${ }^{84}$

The LFER shown in Figure 6 provides evidence of the maturity of computational approaches and their power to operate alongside experimental studies in moving towards a comprehensive explanation of the catalytic rate acceleration for TIM and other enzymes. It has been proposed that the prime imperative for enzymatic catalysis of deprotonation of weak carbon 
acids is that protein catalysts operate on the bound substrates to reduce that thermodynamic driving force for deprotonation of carbon. ${ }^{85-89}$ Figure 6 supports the existence of this imperative, and the LFER provide support for a simple and self-consistent model to rationalize the contribution of individual amino acid side chains to the enzymatic rate acceleration.
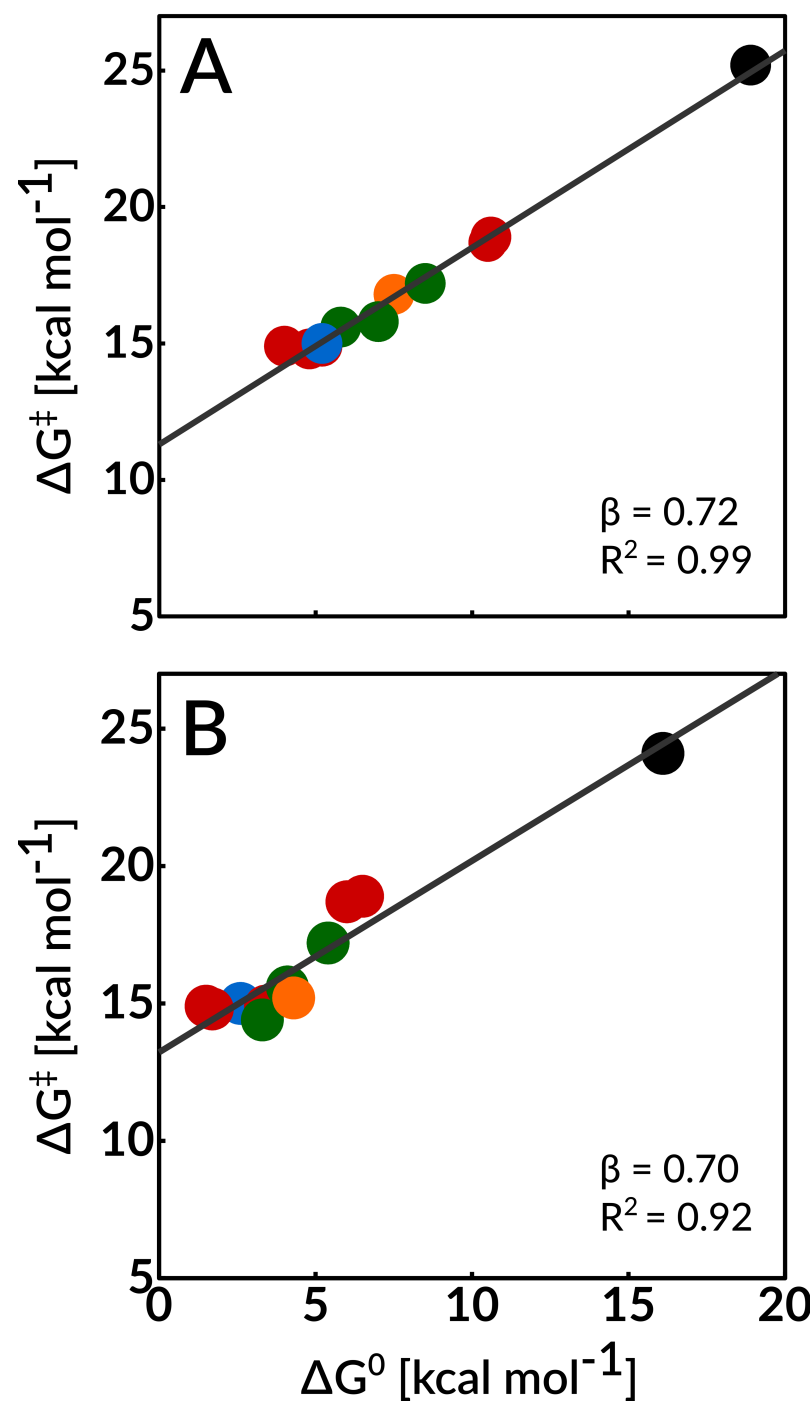

Figure 6. Linear free energy relationships between the activation free energies, $\Delta \mathrm{G}^{*}$, and the reaction free energies, $\Delta \mathrm{G}^{0}$, determined in this work using EVB simulations, for the deprotonation of DHAP and GAP catalyzed by wildtype TIM and all TIM variants described in this study. The correlation coefficients were calculated using regression analysis to be 0.99 and 0.92 for the deprotonation of substrates DHAP and GAP respectively. In this figure, the black circles correspond to the non-enzymatic reaction in aqueous solution, the blue circles correspond to the wild- 
type enzyme, the red circles correspond to single substitutions of polar side chains (K12A and E97A), the green circles correspond to single substitutions of nonpolar side chains (P166A, I170A and L230A), and finally, the orange circle corresponds to double substitutions of nonpolar side chains (I170A/L230A). The raw data used for this figure is provided in Table 1.

The amino acid side chains whose variants satisfy the Brønsted relationship from Figure 6 play the common role in catalysis of isomerization of stabilizing the enzyme-bound enediolate phosphate relative to bound substrate. The action of two types of side chains is modeled by this figure. First, the polar side chain cation of K12 favors substrate deprotonation by E165 through direct stabilization of negative charge at the enediolate oxygen of the reaction intermediate. EVB simulations (Table 1) predict that the $\mathrm{K} 12 \mathrm{G}$ substitution chain results in a $3.7 \mathrm{kcal} \mathrm{mol}^{-1}$ increase in $\Delta \mathrm{G}^{\ddagger}$ for deprotonation of DHAP and a smaller $2.5 \mathrm{kcal}^{\mathrm{mol}}{ }^{-1}$ increase in $\Delta \mathrm{G}^{\ddagger}$ for deprotonation of GAP, because the side chain lies closer to the O-2 compared to the O-1 oxyanions formed by deprotonation of DHAP and GAP, respectively (Figure 2). The substitution results in larger 5.3 and 3.4 increases in $\Delta \mathrm{G}^{0}$ for deprotonation of enzyme-bound DHAP and GAP, respectively, because O-2 and O-1 now bear a full negative charge at the enediolate products.

Second, the nonpolar side chains of P166, I170 and L230 favor substrate deprotonation to form the enediolate phosphate trianion through either enhancement of stabilizing interactions between the polar amino acid side chains and negative charge at the enediolate oxygen of this reaction intermediate, or through enhancement of the basicity of E165. There is evidence that the changes in $\Delta \mathrm{G}^{0}$ and $\Delta \mathrm{G}^{\ddagger}$ reflect both type of effects:

The estimated electrostatic contributions from both protein and solvent to the calculated activation free energies for the deprotonation of DHAP and GAP were reported in a previous 
computational study of wild-type and I170A, L230A, and I170A/L230A variants of TIM, where the changes in the total electrostatic interactions are the sum of many small changes in individual interactions, including the electrostatic contributions from N10, K12, H95, S96, and E129. ${ }^{13}$ These computational results are consistent with a small weakening in the electrostatic interactions for these enzyme variants.

The P166A and I170A substitutions result in 0.7 and 2.3 unit decreases, respectively, in the $\mathrm{p} K_{\mathrm{a}}$ for the carboxylic acid side chain of E165 at the complex between TIM and the enediolate phosphate analog PGA. ${ }^{14,15}$ These decreases in $\mathrm{p} K_{\mathrm{a}}$ are proposed to result from change in the solvation of the carboxylate discussed above, that favors the calculated increases in $\Delta \mathrm{G}^{0}$ for proton transfer at these TIM variants (Figure 6).

\section{Enzymatic Rate Acceleration}

The chemical reaction mechanism for TIM-catalyzed deprotonation of the $\alpha$-carbonyl carbon is not significantly different from that for the non-enzymatic deprotonation of carbon in water, ${ }^{12}$, 90 except that the catalytic side chains are tethered at a protein binding pocket which is complementary to the transition state for carbon deprotonation. ${ }^{91}$ These side chains are activated in some way for catalysis at the enzyme active site, compared with water: this activation results from the utilization of the phosphodianion binding energy to drive conversion of the flexible open enzyme to the reactive caged enzyme-substrate complex. ${ }^{12,} 20,92-94$ The results from EVB calculations show that cage formation provides a global thermodynamic activation for substrate deprotonation that corresponds to 13.8 and $13.4 \mathrm{kcal} \mathrm{mol}^{-1}$ increases, respectively, in the driving force for proton transfer from bound DHAP and GAP, and that $c a .75 \%$ of this thermodynamic activation (10.4 and $10.3 \mathrm{kcal} \mathrm{mol}^{-1}$ respectively) is expressed at the transition state for substrate deprotonation (Table 1). Figure 6 shows that a large number of active site side chains at TIM 
play a common role in catalysis of the isomerization reactions of DHAP and GAP of stabilizing the enzyme-bound enediolate phosphate relative to bound substrate.

The side chains examined in our studies make two distinct contributions to the thermodynamic/kinetic activation of TIM for deprotonation of bound substrate. First, the transition state for proton transfer is stabilized by 3.7 and $2.5 \mathrm{kcal} \mathrm{mol}^{-1}$ for deprotonation of DHAP and GAP, respectively, through interactions with the cationic side chain of K12. These cations provide no significant stabilization of the transition state for proton transfer in water at $\mathrm{pH} \geq 7$, where there is no significant catalysis of deprotonation of GAP or DHAP by tertiary ammonium cations. ${ }^{90}$ Second, the hydrophobic side chains of P166, I170 and L230 occupy positions that minimize the thermodynamic and kinetic barriers to substrate deprotonation. There is good experimental evidence, described above, that the effect on the reaction driving force is due mainly to the enhancement of the basicity of the carboxylate side chain of E165 at TIM compared to aqueous solution. ${ }^{14,15}$ The effects of the P166A, I170A and L230A substitutions on the activation barriers for deprotonation sum to 3.6 and $6.1 \mathrm{kcal} \mathrm{mol}^{-1}$, respectively, for deprotonation of DHAP and GAP. This serves as an upper limit for the contribution of these side chains to catalysis in the event that the effects of these mutations are additive.

There is additional stabilization of the transition state for formation of the enediolate phosphate oxyanion by interactions with other polar side chains and backbone amides at the enzyme active site, most prominently the imidazole side chain of $\mathrm{H} 95,95,96$ and the amide side chain of $\mathrm{N} 10 .{ }^{80}$ These interactions were evaluated as -0.6 (DHAP) and $-1.1 \mathrm{kcal} \mathrm{mol}^{-1}$ (GAP) for H95 and -0.2 (DHAP) and $-1.1 \mathrm{kcal} \mathrm{mol}^{-1}$ (GAP) for N10, obtained by applying the linear response approximation (LRA $)^{74,75}$ to calculated EVB trajectories for the deprotonation of these substrates by wild-type TIM (Figure 4 and Table S6 in the Supporting Information). By 
comparison, the H95Q mutation results in a $c a .3 \mathrm{kcal} \mathrm{mol}^{-1}$ increase in the activation barrier for TIM-catalyzed isomerization. ${ }^{97}$

In conclusion, the results of experimental and computational studies are converging to provide a clear rationale for the $10 \mathrm{kcal} \mathrm{mol}^{-1}$ stabilization of the transition state for deprotonation of TIM-bound substrate (Table 1). The total transition state stabilization represents the combined contributions of many different side chains. The precision in their placement $^{78,98}$ at the TIM active site is the end product of the evolution of a catalytic proteinsubstrate cage that effects a large reduction in the thermodynamic driving force for proton transfer to the carboxylate side chain of E165, ${ }^{14,} 15$ and which is strongly stabilized by interactions with the substrate dianion..$^{20}$

\section{Concluding Remarks}

We present the results of the most extensive computational study of the effect of substitution of key active sites residues on the activity of triosephosphate isomerase reported to date. These data provide biochemical insight into the mode of enzyme action, while probing the limits of the EVB approach in modeling this deceptively simple proton transfer reaction. The EVB simulations reproduce both the changes in enzyme structure determined for the P166A, ${ }^{40}$ I170A, ${ }^{43}$ and $\mathrm{L} 230 \mathrm{~A}^{43} \mathrm{TIM}$ variants, and the changes in the experimental activation free energies

for the broad range of protein variants reported in Table 1. In addition, the simulations provide thermodynamic barriers to substrate deprotonation, that were used in the construction of linear free energy relationships with excellent linear correlations between the kinetic and thermodynamic barriers for the deprotonation of substrates DHAP and GAP over a 10.4 and 14.9 $\mathrm{kcal} \mathrm{mol}^{-1}$ range in activation and reaction free energies respectively. This extended LFER 
(Figure 6) shows that the evolution of the remarkable catalytic proficiency of TIM was driven by the selection of caged enzyme-substrate active site structures that minimize the thermodynamic barrier for the highly unfavourable substrate deprotonation.

The activation barrier for non-enzymatic reactions, which proceed though unstable carbocation (e.g. isopentenyl pyrophosphate isomerase $\mathrm{e}^{99}$ or diterpene synthase ${ }^{100}$ ) or carbanion ${ }^{87}$, 101 reaction intermediates, is composed mainly of the thermodynamic barrier to formation of the intermediate. ${ }^{85}, 102,103 \mathrm{We}$ propose that the rate acceleration for these enzymes is achieved by reducing this thermodynamic barrier through a mechanism similar to that documented for TIM, where the substrate binds to an open form of the protein catalyst, which then undergoes a ligandgated conformational to create a protein-substrate cage. Substrate ionization at this cage is promoted by positioning relevant amino acid side chains so as to provide optimal stabilization of the charged reaction intermediate, relative to the neutral substrate, through electrostatic interactions with catalytic side chains and backbone amides of opposite polarity. ${ }^{78}$ The enzymatic reaction may also be promoted through creation of interactions that destabilize the ground state, and which are relieved at the transition state, such as desolvation of the carboxylate side chain of E165, which enhances the basicity of this side chain. This model defines the contribution of participating side chains to catalysis as the effect of the active site side chains forming this catalytic cage on the equilibrium constant for conversion of enzyme-bound substrate to the reaction intermediate (Figure 6), and posits that the enzymatic rate accelerations are due mainly to preorganization of polar active-site side chains into positions that provide for optimal stabilization of the enzymatic transition state. ${ }^{78,104}$ 


\section{Acknowledgements}

This work was supported by the Swedish Research Council (VR, grant 2015-04298), the US

National Institutes of Health (GM03597 and GM116921), the Knut and Alice Wallenberg Foundation (Wallenberg Academy Fellowship 2013.0124 and 2018.0140), the Human Frontier Science Program (grant RGP0041/2017) and the Sven and Lilly Lawski Foundation for Natural Sciences Research (fellowship to Y.K.). Finally, computational resources were provided by the Swedish National Infrastructure for Computing (SNIC, 2017/12-11 and 2018/2-3).

\section{References}

1. Alagona, G.; Ghio, C.; Kollman, P. A., The Intramolecular Mechanism for the Second Proton Transfer in Triosephosphate Isomerase (TIM): A QM/FE Approach. J. Comput. Chem. 2003, 24, $46-56$.

2. Alagona, G.; Ghio, C.; Kollman, P. A., Do Enzymes Stabilize Transition States by Electrostatic Interactions or $\mathrm{p} K_{\mathrm{a}}$ Balance: The Case of Triose Phosphate Isomerase (TIM)? $J$. Am. Chem. Soc. 1995, 117, 9855-62.

3. Daggett, V.; Kollman, P. A., Molecular Dynamics Simulations of Active Site Mutants of Triosephosphate Isomerase. Protein Eng. 1990, 3, 677-690.

4. Brown, F. K.; Kollman, P. A., Molecular Dynamics Simulations of "Loop Closing" in the Enzyme Triose Phosphate Isomerase. J. Mol. Biol. 1987, 198, 533-46.

5. Alagona, G.; Ghio, C.; Kollman, P. A., Simple Model for the Effect of the Glu165 $\rightarrow$ Asp165 Mutation on the Rate of Catalysis in Triose Phosphate Isomerase. J. Mol. Biol. 1986, 191, 23-27. 6. Alagona, G.; Desmeules, P.; Ghio, C.; Kollman, P. A., Quantum Mechanical and Molecular Mechanical Studies on a Model for the Dihydroxyacetone Phosphate-Glyceraldehyde Phosphate 
Isomerization Catalyzed by Triose Phosphate Isomerase (TIM). J. Am. Chem. Soc. 1984, 106, 3623-32.

7. Cui, Q.; Karplus, M., Quantum Mechanics/Molecular Mechanics Studies of Triosephosphate Isomerase-Catalyzed Reactions: Effect of Geometry and Tunneling on Proton-Transfer Rate Constants. J. Am. Chem. Soc. 2002, 124, 3093-3124.

8. Cui, Q.; Karplus, M., Triosephosphate Isomerase: A Theoretical Comparison of Alternative Pathways. J. Am. Chem. Soc. 2001, 123, 2284-2290.

9. Neria, E.; Karplus, M., Molecular Dynamics of an Enzyme Reaction: Proton Transfer in TIM. Chem. Phys. Lett. 1997, 267, 23-30.

10. Bash, P. A.; Field, M. J.; Davenport, R. C.; Petsko, G. A.; Ringe, D.; Karplus, M., Computer Simulation and Analysis of the Reaction Pathway of Triosephosphate Isomerase. Biochemistry 1991, 30, 5826-32.

11. Joseph, D.; Petsko, G.; Karplus, M., Anatomy of a Conformational Change: Hinged "Lid" Motion of the Triosephosphate Isomerase Loop. Science 1990, 249, 1425-1428.

12. Richard, J. P., A Paradigm for Enzyme-Catalyzed Proton Transfer at Carbon: Triosephosphate Isomerase. Biochemistry 2012, 51, 2652-2661.

13. Kulkarni, Y. S.; Liao, Q.; Petrović, D.; Krüger, D. M.; Strodel, B.; Amyes, T. L.; Richard, J. P.; Kamerlin, S. C. L., Enzyme Architecture: Modeling the Operation of a Hydrophobic Clamp in Catalysis by Triosephosphate Isomerase. J. Am. Chem. Soc. 2017, 139, 10514-10525.

14. Zhai, X.; Reinhardt, C. J.; Malabanan, M. M.; Amyes, T. L.; Richard, J. P., Enzyme Architecture: Amino Acid Side-Chains That Function To Optimize the Basicity of the Active Site Glutamate of Triosephosphate Isomerase. J. Am. Chem Soc. 2018, 140, 8277-8286. 
15. Malabanan, M. M.; Nitsch-Velasquez, L.; Amyes, T. L.; Richard, J. P., Magnitude and Origin of the Enhanced Basicity of the Catalytic Glutamate of Triosephosphate Isomerase. J. Am. Chem. Soc. 2013, 135, 5978-5981.

16. Richard, J. P., The Enhancement of Enzymic Rate Accelerations by Brønsted Acid-Base Catalysis. Biochemistry 1998, 37, 4305-4309.

17. Feng, D. F.; Cho, G.; Doolittle, R. F., Determining Divergence Times with a Protein Clock: Update and Reevaluation. Proc Natl Acad Sci USA 1997, 94, 13028-13033.

18. Knowles, J. R.; Albery, W. J., Perfection in Enzyme Catalysis: The Energetics of Triosephosphate Isomerase. Acc. Chem. Res. 1977, 10, 105-111.

19. Sterner, R.; Hocker, B., Catalytic Versatility, Stability, and Evolution of the $(\beta / \alpha)_{8}$-Barrel Enzyme Fold. Chem. Rev. 2005, 105, 4038-4055.

20. Richard, J. P., Protein Flexibility and Stiffness Enable Efficient Enzymatic Catalysis. J. Am. Chem. Soc. 2019, 141, 3320-3331.

21. Kollman, P. A.; Daggett, V.; Dang, L. X., The Application of Computational Methods to the Study of Enzyme Catalysis by Triose-Phosphate Isomerase and Stabilities of Variants of Bacteriophage T4 Lysozyme. Ciba Found. Symp. 1991, 161, 91-107.

22. Karplus, M.; Evanseck, J. D.; Joseph, D.; Bash, P. A.; Field, M. J., Simulation Analysis of Triose Phosphate Isomerase: Conformational Transition and Catalysis. Faraday Discuss. 1992, 93, 239-248.

23. Wade, R. C.; Luty, B. A.; Demchuk, E.; Madura, J. D.; Davis, M. E.; Briggs, J. M.; McCammon, J. A., Simulation of Enzyme-Substrate Encounter with Gated Active Sites. Nat. Struct. Mol. Biol. 1994, 1, 65-69. 
24. Åqvist, J.; Fothergill, M., Computer Simulation of the Triosephosphate Isomerase Catalyzed Reaction. J. Biol. Chem. 1996, 271, 10010-10016.

25. Zhang, X.; Harrison, D. H. T.; Cui, Q., Functional Specificities of Methylglyoxal Synthase and Triosephosphate Isomerase: A Combined QM/MM Analysis. J. Am. Chem. Soc. 2002, 124, 14871-14878.

26. Guallar, V.; Jacobson, M.; McDermott, A.; Friesner, R. A., Computational Modeling of the Catalytic Reaction in Triosephosphate Isomerase. J. Mol. Biol. 2004, 337, 227-239.

27. Kurkcuoglu, O.; Jernigan, R. L.; Doruker, P., Loop Motions of Triosephosphate Isomerase Observed with Elastic Networks. Biochemistry 2006, 45, 1173-1182.

28. Wang, M.; Lu, Z.; Yang, W., Nuclear Quantum Effects on an Enzyme-Catalyzed Reaction with Reaction Path Potential: Proton Transfer in Triosephosphate Isomerase. J. Chem. Phys. 2006, $124,124516$.

29. Massi, F.; Wang, C.; Palmer, A. G., Solution NMR and Computer Simulation Studies of Active Site Loop Motion in Triosephosphate Isomerase. Biochemistry 2006, 45, 10787-10794.

30. Hu, H.; Lu, Z.; Yang, W., QM/MM Minimum Free-Energy Path: Methodology and Application to Triosephosphate Isomerase. J. Chem. Theory Comput. 2007, 3, 390-406.

31. Díaz-Vergara, N.; Piñeiro, A., Molecular Dynamics Study of Triosephosphate Isomerase from Trypanosoma Cruzi in Water/Decane Mixtures. J. Phys. Chem. B. 2008, 112, 3529-3539.

32. Cansu, S.; Doruker, P., Dimerization Affects Collective Dynamics of Triosephosphate Isomerase. Biochemistry 2008, 47, 1358-1368.

33. Minini, L.; Álvarez, G.; González, M.; Cerecetto, H.; Merlino, A., Molecular Docking and Molecular Dynamics Simulation Studies of Trypanosoma cruzi Triosephosphate Isomerase 
Inhibitors. Insights into the Inhibition Mechanism and Selectivity. J. Mol. Graph. Model. 2015, $58,40-49$.

34. Amrein, B. A.; Steffen-Munsberg, F.; Szeler, I.; Purg, M.; Kulkarni, Y.; Kamerlin, S. C. L., CADEE: Computer-Aided Directed Evolution of Enzymes. IUCRj 2017, 4, 50-64.

35. Kulkarni, Y. S.; Liao, Q.; Byléhn, F.; Amyes, T. L.; Richard, J. P.; Kamerlin, S. C. L., The Role of Ligand-Driven Conformational Changes in Enzyme Catalysis: Modeling the Reactivity of the Catalytic Cage of Triosephosphate Isomerase. J. Am. Chem. Soc. 2018, 140, 3854-3857.

36. Åqvist, J., Cold Adaptation of Triosephosphate Isomerase. Biochemistry 2017, 56, 41694176.

37. Liao, Q.; Kulkarni, Y.; Sengupta, U.; Petrović, D.; Mulholland, A. J.; van der Kamp, M. W.; Strodel, B.; Kamerlin, S. C. L., Loop Motion in Triosephosphate Isomerase is not a Simple Open and Shut Case. J. Am. Chem. Soc. 2018, 140, 15889-15903.

38. Wierenga, R. K., Triosephosphate Isomerase: A Highly Evolved Biocatalyst. Cell. Mol. Life Sci. 2010, 67, 3961-3982.

39. Warshel, A.; Weiss, R. M., An Empirical Valence Bond Approach for Comparing Reactions in Solutions and in Enzymes. J. Am. Chem. Soc. 1980, 102, 6218-6226.

40. Casteleijn, M. G.; Alahuhta, M.; Groebel, K.; El-Sayed, I.; Augustyns, K.; Lambeir, A. M.; Neubauer, P.; Wierenga, R. K., Functional Role of the Conserved Active Site Proline of Triosephosphate Isomerase. Biochemistry 2006, 45, 15483-15494.

41. Go, M. K.; Koudelka, A.; Amyes, T. L.; Richard, J. P., Role of Lys-12 in Catalysis by Triosephosphate Isomerase: A Two-Part Substrate Approach. Biochemistry 2010, 49, 5377-5389. 
42. Malabanan, M. M.; Koudelka, A. P.; Amyes, T. L.; Richard, J. P., Mechanism for Activation of Triosephosphate Isomerase by Phosphite Dianion: The Role of a Hydrophobic Clamp. J. Am. Chem. Soc. 2012, 134, 10286-10298.

43. Richard, J. P.; Amyes, T. L.; Malabanan, M. M.; Zhai, X.; Kim, K. J.; Reinhardt, C. J.;

Wierenga, R. K.; Drake, E. J.; Gulick, A. M., Structure-Function Studies of Hydrophobic Residues That Clamp a Basic Glutamate Side Chain during Catalysis by Triosephosphate Isomerase. Biochemistry 2016, 55, 3036-3047.

44. Chang, T. C.; Park, J. H.; Colquhoun, A. N.; Khoury, C. B.; Seangmany, N. A.; Schwans, J. P., Evaluating the Catalytic Importance of a Conserved Glu97 Residue in Triosephosphate Isomerase. Biochem. Biophys. Res. Commun. 2018, 505, 492-497.

45. Jogl, G.; Rozovsky, S.; McDermott, A. E.; Tong, L., Optimal Alignment for Enzymatic Proton Transfer: Structure of the Michaelis Complex of Triosephosphate Isomerase at 1.2- $\AA$ Resolution. Proc Natl Acad Sci USA 2003, 100, 50-55.

46. Go, M. K.; Amyes, T. L.; Richard, J. P., Rescue of K12G mutant TIM by $\mathrm{NH}_{4}{ }^{+}$and Alkylammonium Cations: The Reaction of an Enzyme in Pieces. J. Am. Chem. Soc. 2010, 132, $13525-13532$.

47. Lodi, P. J.; Chang, L. C.; Knowles, J. R.; Komives, E. A., Triosephosphate Isomerase Requires a Positively Charged Active site: The Role of Lysine-12. Biochemistry 1994, 33, 28092814.

48. Olivares-Illana, V.; Riveros-Rosas, H.; Cabrera, N.; Tuena De Gómez-Puyou, M.; PérezMontfort, R.; Costas, M.; Gómez Puyou, A., A Guide to the Effects of a Large Portion of the Residues of Triosephosphate Isomerase on Catalysis, Stability, Druggability, and Human Disease. Proteins: Struc. Func. Bioinformat. 2017, 85, 1190-1211. 
49. Samanta, M.; Murthy, M. R. N.; Balaram, H.; Balaram, P., Revisiting the Mechanism of the Triosephosphate Isomerase Reaction: The Role of the Fully Conserved Glutamic Acid 97 Residue. ChemBioChem 2011, 12, 1886-1896.

50. Zhang, Z.; Sugio, S.; Komives, E. A.; Liu, K. D.; Knowles, J. R.; Petsko, G. A.; Ringe, D., Crystal Structure of Recombinant Chicken Triosephosphate IsomerasePhosphoglycolohydroxamate Complex at 1.8-Å Resolution. Biochemistry 1994, 33, 2830-7.

51. Li, L.; Luo, M.; Ghanem, M.; Taylor, E. A.; Schramm, V. L., Second-Sphere Amino Acids Contribute to Transition-State Structure in Bovine Purine Nucleoside Phosphorylase. Biochemistry 2008, 47, 2577-2583.

52. Kursula, I.; Wierenga, R. K., Crystal Structure of Triosephosphate Isomerase Complexed with 2-Phosphoglycolate at 0.83-Å Resolution. J. Biol. Chem. 2003, 278, 9544-9551.

53. Davenport, R. C.; Bash, P. A.; Seaton, B. A.; Karplus, M.; Petsko, G. A.; Ringe, D., Structure of the Triosephosphate Isomerase-Phosphoglycolohydroxamate Complex: An Analog of the Intermediate on the Reaction Pathway. Biochemistry 1991, 30, 5821-5826.

54. Lolis, E.; Petsko, G. A., Crystallographic Analysis of the Complex Between Triosephosphate Isomerase and 2-Phosphoglycolate at 2.5-Å Resolution: Implications for Catalysis. Biochemistry 1990, 29, 6619-6625.

55. Malabanan, M. M.; Amyes, T. L.; Richard, J. P., Mechanism for Activation of Triosephosphate Isomerase by Phosphite Dianion: The Role of a Ligand-Driven Conformational Change. J. Am. Chem. Soc. 2011, 133, 16428-16431.

56. Jorgensen, W. L.; Maxwell, D. S.; Tirado-Rives, J., Development and Testing of the OPLS All-Atom Force Field on Conformational Energetics and Properties of Organic Liquids. J. Am. Chem. Soc. 1996, 118, 11225-11236. 
57. Marelius, J.; Kolmodin, K.; Feierberg, I.; Åqvist, J., Q: A Molecular Dynamics Program for Free Energy Calculations and Empirical Valence Bond Simulations in Biomolecular Systems. $J$. Mol. Graph. Model. 1998, 16, 213-225.

58. Bauer, P.; Barrozo, A.; Purg, M.; Amrein, B. A.; Esguerra, M.; Wilson, P. B.; Major, D. T.; Åqvist, J.; Kamerlin, S. C. L., Q6: A Comprehensive Toolkit for Empirical Valence Bond and Related Free Energy Calculations. SoftwareX 2018, 7, 388-395.

59. Berman, H. M.; Westbrook, J.; Feng, Z.; Gililand, G.; Bhat, T. N.; Weissig, H.; Shindyalov, I. N.; Bourne, P. E., The Protein Data Bank. Nucleic Acids Res. 2000, 28, 235-242.

60. Shapovalov, M. X.; Dubrack Jr., R. L., A Smoothed Backbone-Dependent Rotamer Library for Proteins Derived from Adaptive Kernel Density Estimates and Regressions. Structure 2011, $19,844-858$.

61. Pettersen, E. F.; Goddard, T. D.; Huang, C. C.; Couch, G. S.; Greenblatt, D. M.; Meng, E. C.; Ferrin, T. E., UCSF Chimera - A Visualization System for Exploratory Research and Analysis. J. Comp. Chem. 2004, 25, 1605-1612.

62. Jorgensen, W. L.; Chandresekhar, J.; Madura, J. D., Comparison of Simple Potential Functions for Simulating Liquid Water. J. Chem. Phys. 1983, 79, 926.

63. Warshel, A.; King, G., Polarization Constraints in Molecular Dynamics Simulations of Aqueous Solutions: The Surface Constraint All Atom Solvent Model. Chem. Phys. Lett. 1985, $121,124-129$.

64. Olsson, M. H. M.; Søndergaard, C. R.; Rostkowski, M.; Jensen, J. H., PROPKA3: Consistent Treatment of Internal and Surface Residues in Empirical $\mathrm{p} K_{\mathrm{a}}$ Predictions. J. Chem. Theory Comput. 2011, 7Th 525-537. 
65. Shurki, A.; Derat, E.; Barrozo, A.; Kamerlin, S. C. L., How Valence Bond Theory can Help You Understand Your (Bio)chemical Reaction. Chem. Soc. Rev. 2015, 44, 1037-1052.

66. Humphrey, W.; Dalke, A.; Schulten, K., VMD: Visual Molecular Dynamics. J. Mol. Graph. 1996, $14,33-38$.

67. Daura, X.; Gademann, K.; Jaun, B.; Seebach, D.; van Gunsteren, W. F.; Mark, A. E., Peptide Folding: When Simulation Meets Experiment. Angew. Chem. Int. Ed. 2004, 38, 236-240. 68. Pronk, S.; Páll, S.; Schulz, R.; Larsson, P.; Bjelkmar, P.; Apostolov, R.; Shirts, M. R.; Smith, J. C.; Kasson, P. M.; van der Spoel, D.; Hess, B.; Lindahl, E., GROMACS 4.5: A HighThroughput and Highly Parallel Open Source Molecular Simulation Toolkit. Bioinformatics 2013, 29, 845-854.

69. PyMOL The PyMOL Molecular Graphics System, Version 2.0 Schrödinger, LLC.

70. Malabanan, M. M.; Go, M. K.; Amyes, T. L.; Richard, J. P., Wildtype and Engineered Monomeric Triosephosphate Isomerase from Trypanosoma brucei: Partitioning of Reaction Intermediates in $\mathrm{D}_{2} \mathrm{O}$ and Activation by Phosphite Dianion. Biochemistry 2011, 50, 5767-5779.

71. Zhai, X.; Amyes, T. L.; Richard, J. P., Role of Loop-Clamping Side Chains in Catalysis by Triosephosphate Isomerase. J. Am. Chem. Soc. 2015, 137, 15185-15197.

72. Zhai, X.; Go, M. K.; Donoghue, A. C.; Amyes, T. L.; Pegan, S. D.; Wang, Y.; Loria, J. P.; Mesecar, A. D.; Richard, J. P., Enzyme Architecture: The Effect of Replacement and Deletion Mutations of Loop 6 on Catalysis by Triosephosphate Isomerase. Biochemistry 2014, $53,3486-3501$.

73. Joseph-McCarthy, D.; Lolis, E.; Komives, E. A.; Petsko, G. A., Crystal Structure of the K12M/G15A Triosephosphate Isomerase Double Mutant and Electrostatic Analysis of the Active Site. Biochemistry 1994, 33, 2815-2823. 
74. Lee, F. S.; Chu, Z. T.; Bolger, M. B.; Warshel, A., Calculations of Antibody-Antigen Interactions: Microscopic and Semi-Microscopic Evaluation of the Free Energies of Binding of Phosphorylcholine Analogs to McPC603. Protein Eng., Des. Sel. 1992, 5, 215-228.

75. Muegge, I.; Tao, H.; Warshel, A., A Fast Estimate of Electrostatic Group Contributions to the Free Energy of Protein-Inhibitor Binding. Protein Eng., Des. Sel. 1997, 10, 1363-1372.

76. Warshel, A., Energetics of Enzyme Catalysis. Proc Natl Acad Sci USA 1978, 75, 5250-5254.

77. Warshel, A., Electrostatic Origin of the Catalytic Power of Enzymes and the Role of Preorganized Active Sites. J. Biol. Chem. 1998, 273, 27035-27038.

78. Warshel, A.; Sharma, P. K.; Kato, M.; Xiang, Y.; Lui, H.; Olsson, M. H. M., Electrostatic Basis for Enzyme Catalysis. Chem. Rev. 2006, 106, 3210-3235.

79. Fothergill-Gilmore, L. A.; Michels, P. A. M., Evolution of Glycolysis. Prog. Biophys. Molec. Biol 1993, 59, 105-235.

80. Richard, J. P.; Zhai, X.; Malabanan, M. M., Reflections on the Catalytic Power of a TIMbarrel. Bioorg. Chem. 2014, 57, 206-212.

81. Zhai, X.; Amyes, T. L.; Wierenga, R. K.; Loria, J. P.; Richard, J. P., Structural Mutations That Probe the Interactions between the Catalytic and Dianion Activation Sites of Triosephosphate Isomerase. Biochemistry 2013, 52, 5928-5940.

82. Malabanan, M. M.; Amyes, T. L.; Richard, J. P., A Role for Flexible Loops in Enzyme Catalysis. Curr. Opin. Struct. Biol. 2010, 20, 702-710.

83. Lolis, E.; Albert, T.; Davenport, R. C.; Rose, D.; Hartman, F. C.; Petsko, G. A., Structure of Yeast Triosephosphate Isomerase at $1.9 \AA$ Resolution. Biochemistry 1990, 29, 6609-6618. 
84. Belasco, J. G.; Knowles, J. R., Direct Observation of Substrate Distortion by Triosephosphate Isomerase using Fourier Transform Infrared Spectroscopy. Biochemistry 1980, $19,472-477$.

85. Zhai, X.; Malabanan, M. M.; Amyes, T. L.; Richard, J. P., Mechanistic Imperatives for Deprotonation of Carbon Catalyzed by Triosephosphate Isomerase: Enzyme Activation by Phosphite Dianion. J. Phys. Org. Chem. 2014, 27, 269-276.

86. Tsang, W.-Y.; Wood, B. M.; Wong, F. M.; Wu, W.; Gerlt, J. A.; Amyes, T. L.; Richard, J. P., Proton Transfer from C-6 of Uridine 5'-Monophosphate Catalyzed by Orotidine 5'Monophosphate Decarboxylase: Formation and Stability of a Vinyl Carbanion Intermediate and the Effect of a 5-Fluoro Substituent. J. Am. Chem. Soc. 2012, 134, 14580-14594.

87. Richard, J. P.; Amyes, T. L., Proton Transfer at Carbon. Curr. Opin. Chem. Biol. 2001, 5, 626-633.

88. Amyes, T. L.; Richard, J. P., Determination of the $\mathrm{p} K_{\mathrm{a}}$ of Ethyl Acetate: Brønsted Correlation for Deprotonation of a Simple Oxygen Ester in Aqueous Solution. J. Am. Chem. Soc. 1996, 118, 3129-3141.

89. Gerlt, J. A.; Gassman, P. G., An Explanation for Rapid Enzyme-Catalyzed Proton Abstraction from Carbon Acids: Importance of Late Transition States in Concerted Mechanisms. J. Am. Chem. Soc. 1993, 115, 11552-11568.

90. Richard, J. P., Acid-Base Catalysis of the Elimination and Isomerization Reactions of Triose Phosphates. J. Am. Chem. Soc. 1984, 106, 4926-36.

91. Pauling, L., The Nature of Forces Between Large Molecules of Biological Interest. Nature 1948, 161, 707-709. 
92. Amyes, T. L.; Richard, J. P., Specificity in Transition State Binding: The Pauling Model Revisited. Biochemistry 2013, 52, 2021-2035.

93. Go, M. K.; Amyes, T. L.; Richard, J. P., Hydron Transfer Catalyzed by Triosephosphate Isomerase. Products of the Direct and Phosphite-Activated Isomerization of $\left[1-{ }^{13} \mathrm{C}\right]-$ Glycolaldehyde in $\mathrm{D}_{2} \mathrm{O}$. Biochemistry 2009, 48, 5769-5778.

94. Amyes, T. L.; Richard, J. P., Enzymatic Catalysis of Proton Transfer at Carbon: Activation of Triosephosphate Isomerase by Phosphite Dianion. Biochemistry 2007, 46, 5841-5854.

95. Lodi, P. J.; Knowles, J. R., Neutral Imidazole is the Electrophile in the Reaction Catalyzed by Triosephosphate Isomerase: Structural Origins and Catalytic Implications. Biochemistry 1991, 30, 6948-6956.

96. Komives, E. A.; Chang, L. C.; Lolis, E.; Tilton, R. F.; Petsko, G. A.; Knowles, J. R., Electrophilic Catalysis in Triosephosphate Isomerase: The Role of Histidine-95. Biochemistry 1991, 30, 3011-3019.

97. Nickbarg, E. B.; Davenport, R. C.; Petsko, G. A.; Knowles, J. R., Triosephosphate Isomerase: Removal of a Putatively Electrophilic Histidine Residue Results in a Subtle Change in Catalytic Mechanism. Biochemistry 1988, 27, 5948-5960.

98. Blomberg, R.; Kries, H.; Pinkas, D. M.; Mittl, P. R. E.; Grütter, M. G.; Privett, H. K.; Mayo, S. L.; Hilvert, D., Precision is Essential for Efficient Catalysis in an Evolved Kemp Eliminase. Nature 2013, 503, 418-421.

99. Jonnalagadda, V.; Toth, K.; Richard, J. P., Isopentenyl Diphosphate Isomerase Catalyzed Reactions in $\mathrm{D}_{2} \mathrm{O}$ : Product Release Limits the Rate of This Sluggish Enzyme-Catalyzed Reaction. J. Am. Chem Soc. 2012, 134, 6568-6570. 
100. Driller, R.; Janke, S.; Fuchs, M.; Warner, E.; Mhashal, A. R.; Major, D. T.; Christmann, M.; Brueck, T.; Loll, B., Towards a Comprehensive Understanding of the Structural Dynamics of a Bacterial Diterpene Synthase During Catalysis. Nat. Commun. 2018, 9, 1-8.

101. Richard, J. P.; Amyes, T. L.; Goryanova, B.; Zhai, X., Enzyme Architecture: On the Importance of Being in a Protein Cage. Curr. Opin. Chem. Biol. 2014, 21, 1-10.

102. Richard, J. P., Enzyme and Coenzyme Reaction Mechanisms: Editorial Overview. Bioorg. Chem. 2014, 57, 169-170.

103. Toteva, M. M.; Richard, J. P., Mechanistic Imperatives for the Reaction Catalyzed by Isopentenyl Pyrophosphate Isomerase: Free Energy Profile for Stepwise Isomerization in Water through a Tertiary Carbocation Intermediate. Bioorg. Chem. 1997, 25, 239-245.

104. Warshel, A.; Sharma, P. K.; Kato, M.; Parson, W. W., Modeling Electrostatic Effects in Proteins. Biochim. Biophys. Acta - Prot. Proteom. 2006, 1764, 1647-1676. 
Table of Contents Graphic

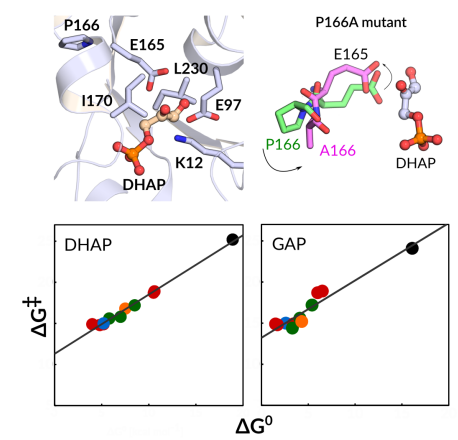

